\title{
TEMPERATURE DAMPING OF PLASMONS IN NONPARABOLIC BAND*
}

\author{
Stefan Goettig \\ Institute of Physics, Warsaw University of Technology, Koszykowa 75, \\ 00-662 Warszawa, Poland \\ (Received August 8, 1990)
}

\begin{abstract}
Damping of plasmons in the nonparabolic free-carrier band of a semiconductor is considered within RPA. Due to the nonparabolic band shape the temperature broadening of plasmons is strongly reduced and even at high temperatures plasmons with large wavevectors may exist as undamped excitations.
\end{abstract}

PACS numbers: $71.45 . \mathrm{Gm}$

Collective excitations influence many effects observed in quantum free-carrier semiconductor plasmas, like, for example, the impurity scattering of carriers for radiation frequencies close to the plasma frequency. Recent studies on linear-conduction-band materials $[1,2]$ indicate that the free-carrier band nonparabolicity may have a strong impact on the plasmons behaviour.

In the present paper the influence of the nonparabolic structure of the free-carrier band on the dispersion of plasmons and their temperature damping is considered. The temperature-dependent formfactor $-\operatorname{Im}[1 / \varepsilon(q, \omega)]$, where $\varepsilon(q, \omega)$ is the wavevector- and frequency-dependent RPA [3] longitudinal complex dielectric function, is used to examine the properties of the collective excitations. The nonparabolic energy band of the form $E(k)=(1 / 2)\left[\left(E_{\mathrm{g}}^{2}+4 P^{2} k^{2}\right)^{1 / 2}-E_{\mathrm{g}}\right]$ is assumed and the overlap integrals of the electronic wave functions calculated from the Kane model are taken into account in the free-carrier polarizability. The calculations were performed for the plasma in $n$-PbSe in isotropic approximation [4], with $P^{2}=\hbar^{2} E_{\mathrm{g}} / 2 m^{*}$, where $E_{\mathrm{g}}$ is the energy gap and $m^{*}$ is the effective mass at the bottom of the conduction band. The dielectric function included also the optical-phonon contribution [e.g. 4] i.e. we account for the plasmon-phonon coupling.

For the degenerate plasma the nonparabolicity of the free-carrier band was found to extend the range of the wavevectors and energies where the plasmons

*This work was supported in part by CPBP 01.03. 


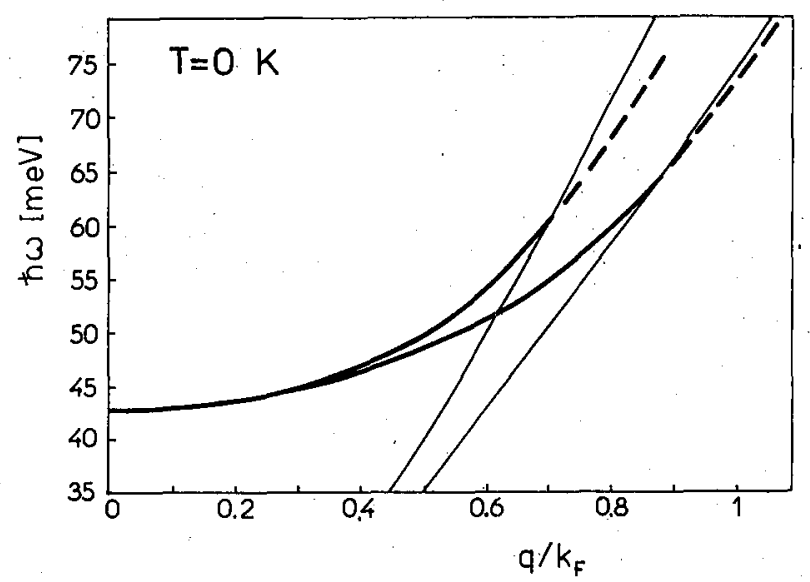

Fig. 1. Plasmon dispersion at $T=0$ in PbSe with $n=2 \times 10^{18} \mathrm{~cm}^{-3}$; notation: upper thick curve - parabolic plasma, lower thick curve - nonparabolic plasma, solid parts of the thick curves - undamped plasmons, broken parts of the thick curves - damped plasmons, thin curves - single-particle excitation boundaries for the nonparabolic (right) and parabolic (left) plasma.

are well defined elementary excitations. This is shown in Fig. 1 where the plasmon dispersion at $T=0 \mathrm{~K}$ is shown for PbSe with the carrier concentration $n=$ $2 \times 10^{18} \mathrm{~cm}^{-3}$. The plasmon energies for the nonparabolic and parabolic plasma are shown by the thick lower and upper curves, respectively. The solid parts of the dispersion curves represent undamped plasmons which became Landau-damped (broken curves) after crossing the apppropriate single-particle excitation (or $\operatorname{Im}[\varepsilon(q, \omega)] \neq 0)$ boundaries indicated by the respective thin lines. Dispersion curves were obtained from the maxima of the formfactor and the wavevector is measurred in the Fermi momentum $k_{\mathrm{F}}$ units.

At high temperatures the influence of the band nonparabolicity is more profound. Namely, in the case of plasmons from the parabolic band their temperature damping, although weak at small $\boldsymbol{q}$, is present for all plasmon wavelengths. In the nonparabolic case, however, even at high temperatures, we have found the plasmons to exist as strictly undamped excitations (within the considered RPA) up to quite large wavevectors. This is illustrated in Fig. 2 where the plasmon dispersion at $T=300 \mathrm{~K}$ is shown for the plasma in PbSe with the carrier concentration $n=2 \times 10^{18} \mathrm{~cm}^{-3}$. The upper thick broken curve corresponds to the temperature-damped plasmon for the parabolic band. The lower thick solid curve corresponds to the nonparabolic plasmon which is undamped until the dispersion curve crosses (at $q=q_{c}$ ), the boundary of single-particle excitations (i.e. the boundary of $\operatorname{Im}[\varepsilon(q, \omega)] \neq 0$. The boundary, indicated by the thin broken line, is (for $\left.\hbar \omega<E_{\mathrm{g}}\right)$ given by the equation $\hbar \Omega(q)=\left(E_{0} E_{\mathrm{g}}\right)^{1 / 2}\left(q / k_{\mathrm{F}}\right)$, where $E_{0}=\hbar^{2} k_{\mathrm{F}}^{2} / 2 m^{*}$. We note that the sharp single-particle boundary at $T>0$ is de- 


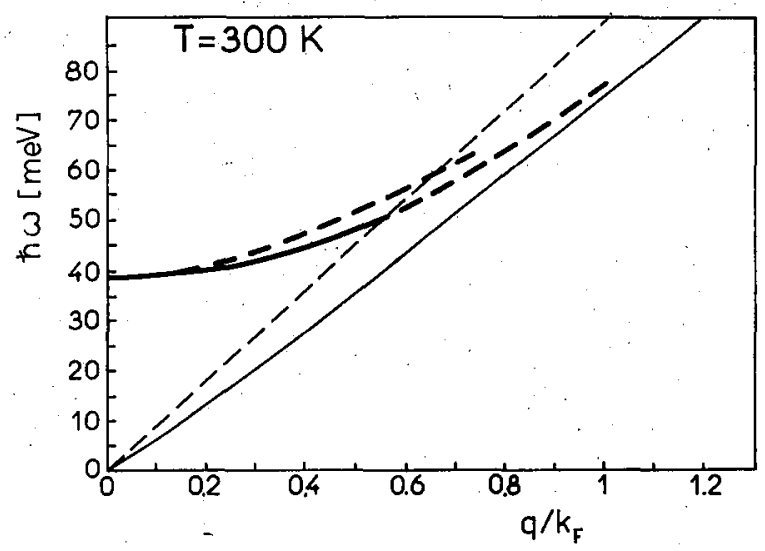

Fig. 2 Plasmon dispersion at $T=300 \mathrm{~K}$ in PbSe with $n=2 \times 10^{18} \mathrm{~cm}^{-3}$; notation: upper thick broken curve - parabolic plasma, damped plasmon; lower thick curve nonparabolic plasma, with solid part repesenting the undamped plasmon and broken part representing the damped plasmon; thin lines - boundaries of single-particle excitations in the nonparabolic plasma at $T>0$ (broken) and $T=0$ (solid).

termined solely by the assumed nonparabolic shape $E(k)$ of the free-carrier band, being independent of the particular form of the overlap integrals. In the limit of the parabolic band the boundary coincides with the vertical axes i.e. for the parabolic band plasmons are damped for all $T>0$. The single-particle boundary for $T=0 \mathrm{~K}$ is also shown in Fig. 2 for comparison. For wavevectors $q>q_{c}$ the usual temperature broadening of the nonparabolic plasmons occurs, with the plasmon peak in the formfactor decaying with increasing $q$. The comparison of the temperature broadening of the plasmon peaks for the nonparabolic and parabolic band, shows that plasmons in the nonparabolic band may be recognized as elementary excitations up to much larger values of $q$ than in the parabolic band. This is indicated in Fig. 2 by the length of the dispersion curves penetrating the $\operatorname{Im}[\varepsilon(q, \omega)] \neq 0$ region.

In conclusion, we found that the nonparabolic shape of the free-carrier band strongly reduces the damping of plasmons, leading in particular to the existence of undamped collective excitations at arbitrary high temperatures.

\section{References}

[1] Q. Dingrong, W. Szuszkiewicz, Z. Jiaming, W. Bardyszewski, in Proc. 19th Internat. Conf. on the Physics of Semiconductors, Warsaw 1988, ed. W. Zawadzki, Institute of Physics, Polish Academy of Sciences, Warsaw 1988, p. 1715.

[2] W. Bardyszewski, W. Szuszkiewicz, Q. Dingrong, Z. Jiaming, Acta Phys. Pol. A77, 269 (1990). 
[3] H. Ehrenreich, M.H. Cohen, Phys. Rev. 115, 786 (1959).

[4] S. Goettig, J. Phys. C, Solid State Phys. 13, 1751 (1981). 